\title{
Percepção do agente comunitário de saúde sobre qualidade de vida e qualidade de vida no trabalho
}

\author{
Perception of the community health agent on quality \\ of life and quality of life at work
}

Resumo O Agente Comunitário de Saúde (ACS), profissional integrante da equipe mínima da Estratégia Saúde da Família (ESF), desenvolve seu trabalho com um olhar voltado para o processo saúde-doença-cuidado das famílias, sujeitos e comunidades, num território adscrito, com o intuito da melhoria da qualidade de vida destes. O estudo objetivou identificar o conhecimento do ACS acerca da qualidade de vida e qualidade de vida no trabalho e descrever o perfil sociodemográfico das famílias, sujeitos e comunidades. Estudo exploratório-descritivo, com abordagem qualitativa, desenvolvida com cinco ACS do território da ESF de Alto dos Honório, zona Rural de Cariré-Ceará, durante o período de março a maio de 2013, a partir de um questionário. De forma singular, existe a compreensão de que a qualidade de vida está diretamente relacionada às escolhas dos hábitos de vida mais saudáveis, associadas à sensação de segurança por sentir-se cuidado pela equipe da ESF e por ter a condição de cuidar da população sob sua responsabilidade. Os resultados apontam que os ACS compreendem parcialmente os conceitos de qualidade de vida e qualidade de vida no trabalho, associando estes a hábitos de vida saudáveis, bem como, à realização de suas práticas laborais com afeto e responsabilidade. Palavras-chave: Agentes Comunitários de Saúde. Trabalho. Qualidade de Vida. Prática Profissional.

Francisco Rosemiro Guimarães Ximenes Neto ${ }^{\mathrm{I}}$

Benedita Tatiane Gomes

LIBERATO ${ }^{\text {II }}$

Maria Roselange Guimarães

XIMENES $^{\text {II }}$

Francisco Diógenes dos Santos ${ }^{I I I}$

Karla Mara Coelho Ponte de

Oliveira $^{\mathrm{I}}$

Ana Gerúsia SouZa Ribeiro

GURGEL $^{1}$

${ }^{\mathrm{I}}$ Universidade Estadual Vale do Acaraú (UVA). Sobral/CE - Brasil. "Centro Universitário Inta

(UNINTA). Sobral/CE - Brasil.

IIIEscola de Saúde Pública do Ceará

Paulo Marcelo Martins Rodrigues

(ESP/CE). Fortaleza/CE - Brasil.
Abstract The Community Health Agent (CHA), a professional member of the minimum team of the Family Health Strategy (FHS), develops its work with a view towards the health-illness-care process of families, subjects and communities, in an attached territory, with the aim of improving their quality of life. The study aimed to identify the knowledge of the CHA about quality of life and quality of life at work and to describe the sociodemographic profile of families, subjects and communities. A descriptive, exploratory study with a qualitative approach was developed with five CHA from the FHS in Alto dos Honório, in the rural area of Cariré-Ceará, during the period from March to May 2013, based on a questionnaire. In a singular way, there is an understanding that the quality of life is directly related to the choices of healthier life habits, associated to the feeling of security because of the care taken by the FHS team and to the condition of caring for the population under its responsibility. The results show that CHAs partially understand the concepts of quality of life and quality of life at work, associating them with healthy life habits as well as the accomplishment of their work practices with affection and responsibility.

Keywords: Community Health Agents. Work. Quality of life. Professional Practice. 


\section{INTRODUÇÃO}

Nos anos 1980, o povo brasileiro vivenciou um intenso processo de reabertura política e, por conseguinte, a redemocratização do país, culminando com a promulgação da Constituição Federal. Entre as inúmeras inovações e avanços, nota-se a criação do Sistema Único de Saúde (SUS) que, após sua regulamentação, em 1990, por meio das Leis n. 8.080/1990 e n. $8.142 / 1990$, foi incrementado o processo de descentralização administrativo-financeira, implantando políticas, programas, projetos, serviços e ações de saúde. ${ }^{1}$

O Ministério da Saúde criou, em 1991, o Programa Nacional de Agentes Comunitários de Saúde (PNACS), como mais uma entre as estratégias de busca pela mudança do modelo assistencial vigente, reorganização da atenção à saúde e descentralização da Atenção Primária à Saúde (APS). O PNACS, posteriormente, em 1992, passou a ser denominado pela comunidade sanitária de Programa de Agentes Comunitários de Saúde (PACS). O PACS advém de experiências localizadas, por exemplo, o Ceará o implantou, em $1987,{ }^{2}$ como parte integrante de um programa que procurava contribuir para a geração de emprego em meio à conjuntura de seca e reduzir a mortalidade materna e infantil.

O PACS contribuiu com a melhoria de vários indicadores como os da cobertura vacinal, da redução, devido ao uso de soro caseiro, dos óbitos infantis por diarreias, entre outros. Os avanços do programa demandaram a necessidade de incrementar a descentralização da APS, com maior escala e aporte profissional.
Com isso, o Ministério da Saúde institucionaliza, em março de 1994, o Programa Saúde da Família (PSF), com a concepção de um modelo de atenção à saúde que buscava o desenvolvimento de ações de promoção e proteção à saúde das famílias, dos sujeitos e das comunidades, por meio de equipes de saúde, com atendimento em unidade local e na comunidade, no nível da Atenção Primária à Saúde. ${ }^{3}$

Em pouco tempo o PSF avança em números de equipes implantadas em todo o Brasil, além da melhoria dos indicadores de morbidade e mortalidade, principalmente, os maternos e infantis. Contudo, em 1997, como busca de reorientação do modelo assistencial, o PSF passa a ser política governamental, denominada de Estratégia Saúde da Família (ESF). ${ }^{1,4}$ A organização da ESF, desde sua concepção, envolve uma equipe mínima formada por um enfermeiro, um médico, um auxiliar de enfermagem ou técnico de enfermagem e ACS em número suficiente, conforme o perfil do território sanitário. ${ }^{5}$

O ACS, nos territórios, tem atuado junto às famílias, com prioridade para gestantes, crianças, idosos, sujeitos com doenças crônicas transmissíveis e não transmissíveis e em situação de risco e/ou vulnerabilidade. Contudo, o ACS desenvolve seu processo de trabalho, em muitos casos, além das 40 horas de trabalho exigidas, por conta da diversidade do território, seus determinantes e perfil epidemiológico, o que exige uma maior dedicação, tempo e esforço, podendo afetar sua qualidade de vida no trabalho e no lado pessoal. A partir de tal contexto, faz-se o seguinte questionamento: O que o ACS entende por qualidade de vida e qualidade de vida no trabalho? 
Assim, o estudo tem por objetivo identificar o conhecimento do ACS acerca da qualidade de vida e qualidade de vida no trabalho e na comunidade.

\section{MÉTODo}

A pesquisa foi realizada a partir de um estudo exploratório-descritivo, com abordagem qualitativa, desenvolvido com cinco ACS do território da ESF de Alto dos Honório, zona rural do município de Cariré-Ceará, durante o período de março a maio de 2013.

O município de Cariré está localizado a Noroeste do Estado do Ceará, na Microrregião de Sobral, com uma área de 756.893 $\mathrm{km}^{2}$, distante $287,1 \mathrm{~km}$ de Fortaleza; localizado no semiárido, com uma população de 19.354 habitantes, e uma densidade demográfica de 26,7 habitantes. As principais rendas do município derivam da agricultura de subsistência, pecuária extensiva, emprego público, aposentadoria e programas assistenciais do Governo Federal, por exemplo, o Programa Fome Zero. Quanto à estrutura organizativa da APS/ESF, Cariré possui nove equipes da ESF credenciadas e implantadas, 41 ACS e sete equipes de Saúde Bucal. ${ }^{6}$

A equipe da ESF do território de Alto dos Honório é composta por uma enfermeira, uma técnica de enfermagem, uma cirurgiã-dentista, uma técnica em saúde bucal, um motorista, um atendente de farmácia, duas auxiliares de serviços gerais, dois zeladores de patrimônio e três orientadores sanitários e cinco ACS, atendendo às comunidades de Alto dos Honório, Santana, Caveira, Floresta, Muquém, Muquém de Dentro e Rosilha. ${ }^{7}$
Para preservação da identidade e integridade dos sujeitos, o nome das ACS foi substituído pela sigla ACS acompanhados de um numeral arábico. Os sujeitos do estudo todos são do sexo feminino e apresentam o seguinte perfil:

- ACS 1 - Casada, 41 anos de idade, ensino médio completo, 16 anos que atua na profissão;

- $\quad$ ACS 2 - Casada, não referiu a idade, ensino médio completo, 18 anos que atua na profissão;

- ACS 3 - Casada, 36 anos de idade, ensino médio completo, 15 anos que atua na profissão;

- ACS 4 - Solteira, 55 anos de idade, ensino médio completo, 18 anos que atua na profissão;

- ACS 5 - Casada, 43 anos de idade, ensino médio completo, 17 anos que atua na profissão.

As ACS do estudo têm como salário base o valor de R $\$ 723,01$ (setecentos e vinte e três reais e um centavo), equivalendo a um salário mínimo, acrescidos de meio salário mínimo referente à gratificação estabelecida pelo município.

A coleta das informações foi realizada por meio de um questionário, elaborado pelos pesquisadores, composto por perguntas abertas e fechadas, contendo as seguintes variáveis: perfil e conhecimento acerca da qualidade de vida e qualidade de vida no trabalho. O contato com os sujeitos do estudo para aplicação do questionário ocorreu durante a roda semanal dos ACS com a enfermeira do território. Inicialmente, foram apresentados aos ACS os objetivos do estudo e o Termo de Consentimento Livre e Esclarecido (TCLE). Em seguida, os 
questionários foram entregues para serem preenchidos.

As informações foram sistematizadas e analisadas em categorias e interpretadas com base na literatura pertinente. Quanto às categorias, utilizou-se o referencial de Minayo, ${ }^{8}$ que sugere sua utilização para o estabelecimento de classificações, agrupando elementos, ideias ou expressões em torno de um conceito capaz de abranger tudo isso. Nessa perspectiva, as categorias abstraídas dos discursos dos sujeitos foram: Qualidade de vida, Qualidade de vida no trabalho.

$\mathrm{O}$ estudo observou o emanado pela Resolução n. 466/12, de 12 de dezembro de 2012, que dispõe sobre as Diretrizes e Normas Reguladoras de Pesquisa Envolvendo Seres Humanos do Conselho Nacional de Saúde (CNS), enfatizando os compromissos éticos para com os sujeitos da pesquisa, seja individual ou coletivamente, respeitando os princípios da beneficência, autonomia, não maleficência e justiça. ${ }^{9}$ Vale ressaltar que o estudo foi submetido ao Comitê de Ética e Pesquisa (CEP) da Universidade Estadual Vale do Acaraú (UVA), obtendo parecer favorável à sua realização de n. 681 .

\section{RESULTADOS E DISCUSSÃo}

Os resultados estão expostos em duas categorias analíticas, que foram abstraídas das falas dos sujeitos.

\section{QUALIDADE DE VIDA}

Alimentação saudável; participação na unidade de saúde; participar dos grupos que foi convidada; fazer exercício físico, como caminhada e alongamento; sorrir, amar e ser feliz (ACS 1).
É ter saúde e uma boa alimentação (ACS 2).

É está bem de saúde e consigo mesmo. Ter uma boa alimentação, fazer esporte, se divertir, brincar e está bem com sua família, seus amigos e trabalho (ACS 3).

Ao meu entender: com saúde; ter um acompanhamento médico que merecemos; ter material para trabalharmos como nossa população merece; estarmos com um salário que esteja suprindo nossas necessidades (ACS 4).

... acompanhamento amplo e favorável [...]. Trabalharmos com saúde (ACS 5).

A partir das respostas dos ACS, percebe-se que estes compreendem a qualidade de vida sob perspectivas variadas, citando desde a importância de manter uma alimentação saudável e praticar atividades físicas, até a relevância de participar de grupos e outras atividades sociais como as realizadas na unidade de saúde. O acesso ao lazer, a garantia de acompanhamento de saúde, receber um salário digno e disponibilidade de recursos para o desenvolvimento do trabalho também foram destacados.

A Organização Mundial da Saúde (OMS) entende a qualidade de vida como a "percepção do indivíduo a respeito de sua vida, diante dos aspectos culturais e valores em que ele está inserido e em relação aos seus objetivos, expectativas, padrões e preocupações" ${ }^{10}$ O Ministério da Saúde do Brasil, no glossário temático de Economia da Saúde, define qualidade de vida relacionada à saúde como a "condição em que é possível identificar, de forma subjetiva e global, a satisfação de um indivíduo em relação ao seu estado de saúde, com base em critérios fundamentais estipulados no âmbito da saúde". 11 A nova concepção de saúde "importa uma visão afirmativa, que a identifica com bem-estar e qualidade de vida, e não simplesmente com ausência 
de doença. A saúde deixa de ser um estado estático, biologicamente definido, para ser compreendida como um estado dinâmico, socialmente produzido". ${ }^{12}$

Segundo Minayo, Hartz e Buss, ${ }^{13}$ o patamar mínimo para se falar em qualidade de vida diz respeito à satisfação das necessidades mais essenciais da vida do homem, entre elas: alimentação, trabalho, saúde e lazer. Em relação à alimentação, pode-se dizer que ela está diretamente relacionada à qualidade de vida, porque a nutrição depende da boa qualidade dos alimentos consumidos, tanto como fonte de energia para realizar atividades rotineiras, quanto por estar envolvida no desenvolvimento de doenças crônicas, como a obesidade, diabetes mellitus, e a hipertensão arterial sistêmica.

No caso do trabalho, vale ressaltar que se configura em um espaço privilegiado que também se relaciona à qualidade de vida, pois a satisfação pessoal só pode ser alcançada quando se trabalha com subsídios necessários para o pleno desenvolvimento de suas tarefas e quando se obtém um reconhecimento do desempenho profissional por meio de incentivos financeiro, educacional e social.

No caso dos ACS, cabe citar, ainda, a participação ativa em grupos e em outras atividades que são desenvolvidas na própria unidade de saúde, já que seu trabalho é abrangente/diversificado e a sua qualidade de vida está relacionada à pluralidade das ações que são capazes de executar com acreditação e entusiasmo.

Outra resposta apontada pelos ACS como fator relacionado à qualidade de vida foi o lazer. Este é uma boa argumentação para uma melhor qualidade de vida, justamente porque trata do prazer em realizar algo, isso traz bons motivos para acreditar que a qualidade de vida e o lazer estão relacionados. ${ }^{13}$

Já em relação à prática de atividade física, Ursine et al. ${ }^{14}$ afirmam que ela se relaciona à qualidade de vida, destacando sua efetividade na prevenção de doenças crônicas não transmissíveis e na prevenção de incapacidade devido a estas. Assim, os ACS, apesar de desenvolverem suas atividades, na maior parte do tempo em suas microáreas, em visita domiciliar, busca ativa, campanhas e cadastramento de famílias, percorrendo grandes distâncias caminhando, o que gera um casto calórico relacionado ao trabalho, ${ }^{15}$ se limitam ao não desenvolvimento de outro tipo de atividade física e/ou atitudes saudáveis, a exemplo uma alimentação "adequada", sendo assim sedentários.

O sedentarismo é identificado, atualmente, como um importante problema de saúde pública, tento em vista os fatores de risco para agravos à saúde que desencadeia, a exemplo doenças cardiovasculares. Entre os profissionais da saúde, em especial os ACS, a falta de atividade física e adoção de atitudes saudáveis se torna ainda mais preocupante, uma vez que estes detêm o papel de educar e orientar a população quanto à adoção de atitudes saudáveis, possibilitando a modificação e a melhoria de comportamentos acerca de um estilo de vida saudável. ${ }^{16}$

Logo, faz-se necessário que os ACS sejam estimulados a incorporar, durante suas rotinas, atitudes saudáveis, como a prática de exercícios físicos, além da caminhada já realizada durante o trabalho; alimentação saudável, entre outras, já que são agentes de transformação, de referência para as famílias, indivíduos e comunidades em que atuam. 
Em suma, os conceitos de qualidade de vida referidos pelos ACS tratam de ações que contribuem para a melhoria da sua saúde, do desempenho de seu trabalho e da assistência à comunidade. Desse modo, a qualidade de vida está relacionada a uma gama diversificada de fatores que interferem na sua vida pessoal, no seu ambiente de trabalho e, por consequência, no bem-estar da população a que assistem.

\section{QUALIDADE DE VIDA NO TRABALHO}

\begin{abstract}
É fazer seu trabalho com responsabilidade e gostar de fazê-lo com alegria e prazer (ACS 1).

É ter um vínculo com a sociedade e muito bem informado (ACS 2).

É cumprir o horário de trabalho e termos mais profissionais da saúde [...]. Ser bem recebido no nosso local de trabalho. Receber nosso incentivo em dia e no dia certo. Sermos atendidos quando precisamos, pois, somos seres humanos e também ficamos doentes (ACS 3).

Estarmos com saúde. Termos mais profissionais, como médicos, mais enfermeiros etc. (ACS 4).

Trabalhar com saúde; ter mais profissionais, como por exemplo: um médico, um psicólogo e um terapeuta. E também, um melhor incentivo, no caso do agente que se desloca para cobrir outra área [...] (ACS 5).
\end{abstract}

A qualidade de vida no trabalho abrange dois conceitos opostos, por um lado estão as reivindicações dos colaboradores quanto ao seu bem-estar e satisfação no seu trabalho; por outro está o interesse das organizações quanto à produtividade e qualidade dos bens e serviços. ${ }^{17} \mathrm{~A}$ qualidade de vida no trabalho "está diretamente associada à satisfação dos trabalhadores no desempenho de suas funções". ${ }^{18}$
Os ACS são membros fundamentais na equipe da ESF, pois vivenciam ativamente os problemas de sua comunidade, já que residem na área onde trabalham. Dessa forma, atuam como elo entre a população e os serviços de saúde, sendo algumas de suas funções as visitas aos lares, o cadastramento das famílias, o mapeamento da comunidade e as reuniões comunitárias. ${ }^{5}$

Desse modo, a qualidade de vida no trabalho dos ACS está intimamente relacionada à promoção da saúde da população a que assistem, pois quanto maior seu grau de satisfação profissional, tanto maior sua capacidade de desenvolver seu trabalho com competência e resolubilidade, o que reflete na melhoria das condições de saúde da comunidade.

Ao analisar as respostas dos ACS sobre o conceito de qualidade de vida no trabalho, observa-se que eles possuem pontos de vida variados que englobam definições de responsabilidade no trabalho, gostar e ter prazer em realizar suas atividades, ter um vínculo forte com a comunidade a que assistem, ter acesso à informação, cumprir o horário de trabalho, trabalhar com mais profissionais da saúde, ser bem acolhido no local de trabalho, receber o incentivo em dia e ter direito à assistência de saúde, toda vez que for necessário.

Observa-se que os ACS referem a responsabilidade no trabalho como determinante de sua qualidade de vida, evidenciando a importância do seu compromisso junto à comunidade, pois a responsabilidade social reflete no desenvolvimento pleno de seu trabalho e, por conseguinte, na sua qualidade de vida. 
Identifica-se nas falas dos ACS que a qualidade de vida no trabalho está associada às dificuldades encontradas no dia a dia, como o acesso restrito à informação, que dificulta a realização de suas tarefas diárias; a quantidade insuficiente de outros profissionais da saúde, como médico, enfermeiro e psicólogo, que impede o atendimento qualificado e resolutivo para a população; o acolhimento no ambiente de trabalho ineficaz, que não facilita o acesso da comunidade aos serviços de saúde; o não recebimento do incentivo por desempenho, que pode gerar desestímulo e sentimento de desvalorização do trabalho; a não assistência a sua saúde, quando se encontram em condições vulneráveis ou acometidos por alguma patologia. Essa realidade evidencia que a qualidade de vida no trabalho dos ACS está intimamente relacionada ao suprimento das necessidades que encontram rotineiramente, durante sua jornada de trabalho.

Nesse contexto, Mascarenhas et al. ${ }^{19}$ afirmam que os ACS:

...vêm suportando cargas de trabalho cada vez
maiores, alto grau de frustração e desconten-
tamento em relação à responsabilidade e exer-
cício profissional, exposição a fatores de risco
pertinentes ao ambiente, baixa remuneração,
dificuldades de relacionamento interpessoal,
dentre outros aspectos ocupacionais, o que
pode gerar agravos a sua saúde e prejuízos em
sua qualidade de vida.

Dessa forma, compreender o conceito de qualidade de vida no trabalho pelos ACS se torna um mecanismo de sustentação para a implantação de estratégias que visem a redução do sofrimento destes e a prevenção de riscos, agravos e doenças relacionadas ao trabalho. Pois, um processo de trabalho que seja embasado numa boa qualidade de vida no trabalho corroborará com o alcance da integralidade da atenção e, por conseguinte, com a satisfação da clientela e melhoria da qualidade de vida da população. ${ }^{20}$

\section{CONSIDERAÇões FINAIS}

Acredita-se que conceituar qualidade de vida seja um processo muito mais vivencial do que teórico-científico, pois se inicia a partir da condição e do local em que cada ser humano está; das experiências, relações e efeitos que se têm a partir de um referencial que proporcione satisfação das necessidades.

Os resultados apontam que os ACS compreendem parcialmente os conceitos de qualidade de vida, associando-os a ações que contribuem para a melhoria da sua saúde, de seu desempenho no trabalho e da assistência às famílias e comunidade.

Fica evidente, nas falas dos ACS, que a responsabilidade no trabalho é determinante para a sua qualidade de vida no trabalho, evidenciando o seu compromisso junto à comunidade e sua responsabilidade social refletida na busca por um desenvolvimento pleno de seu trabalho.

Destaca-se, como fator limitante do estudo, a escassez de estudos abordando esta temática, o que nos remete para a necessidade de aprofundar o debate por meio de novos estudos. Diante dessa realidade, constamos a necessidade de extensa e profunda reflexão acerca da qualidade de vida e qualidade de vida no trabalho dos ACS, uma vez que ambas as qualidades influenciam diretivamente na qualidade da assistência prestada às famílias-sujeitos-comunidades, promovendo mudanças nos serviços de saúde e garantindo condições de trabalho mais adequadas. 


\section{REFERÊNCIAS}

1. Ximenes Neto FRG. Gerenciamento do território na Estratégia Saúde da Família: o processo de trabalho dos gerentes [dissertação]. Fortaleza: Universidade Estadual do Ceará-UECE; 2007 [Internet]. Acesso em: 2017 jul. 17. Disponível em: http://www.uece.br/cmasp/dmdocuments/ rosemiro 2007.pdf.

2. Silva MJ. Agente de Saúde: agente de mudança? A experiência do Ceará. Fortaleza: Pós-graduação/DENF/UFC/Fundação Cearense de Pesquisa e Cultura, 1997.

3. Brasil. Ministério da Saúde. Fundação Nacional de Saúde. Departamento de Operações. Coordenação de Saúde da Comunidade. Programa Saúde da Família: saúde dentro de casa. Brasília: Ministério da Saúde, 1994 [Internet], 1994. Acesso em: 2017, dez. 12. Disponível em: http:// bvsms.saude.gov.br/bvs/publicacoes/saude dentro casa programa saude familia.pdf.

4. Brasil. Ministério da Saúde. Secretaria de Assistência à Saúde. Coordenação de Saúde da Comunidade. Saúde da Família: uma estratégia para a reorientação do modelo assistencial. Brasília: Ministério da Saúde, 1997 [Internet]. Acesso em: 2017, ago. 30. Disponível em: http://bvsms. saude.gov.br/bvs/publicacoes/cd09 16.pdf

5. Brasil. Ministério da Saúde. Secretaria de Atenção à Saúde. Departamento de Atenção Básica. Política Nacional de Atenção Básica. Brasília: Ministério da Saúde, 2012 [Internet]. Acesso em: 2017, ago. 19. Disponível em: http://189.28.128.100/dab/docs/publicacoes/geral/pnab.pdf.

6. Ximenes Neto FRG, Martins FR, Aguiar DT, Liberato BTG. Plano Municipal da Saúde, 2010 a 2013: caminhos para um município saudável. Biblioteca Lascasas, 2015, 11(2) [Internet], 2015. Acesso em: 2017, dez. 19. Disponível em: http://www.index-f.com/lascasas/documentos/lc0824.php.

7. Cariré. Secretaria da Saúde. Sistema de Informação da Atenção Básica-SIAB. Relatório equipe da Estratégia Saúde da Família de Alto dos Honório. Cariré: Secretaria da Saúde, 2016.

8. Minayo MCS. O Desafio do Conhecimento: pesquisa qualitativa em saúde, 14. ed. São Paulo: HUCITEC, 2014.

9. Brasil. Ministério da Saúde. Conselho Nacional de Saúde. Resolução n. 466, de 12 de dezembro de 2012 [Internet], 2012. Acesso em: 2017, dez. 19. Disponível em: http://conselho.saude.gov.br/ resolucoes/2012/Reso466.pdf.

10. The WHOQOL Group. The World Health Organization quality of life assessment (WHOQOL): position paper from the World Health Organization. Soc Sci Med. [Internet], 1995. Acesso em: 2017, set. 15, 41: 1.403-10. Disponível em: http://www.sciencedirect.com/science/article/ pii/027795369500112K.

11. Brasil. Ministério da Saúde. Secretaria-Executiva. Secretaria de Ciência, Tecnologia e Insumos Estratégicos. Glossário temático: economia da saúde, 2. ed. amp. Brasília: Editora do Ministério da Saúde, 2009, 60 p. (Série A. Normas e Manuais Técnicos.) [Internet]. Acesso em: 2017, mai. 12. Disponível em: http://bvsms.saude.gov.br/bvs/publicacoes/glossario ecos2.pdf.

12. Buss PM. Promoção da saúde e qualidade de vida. Ciênc. saúde coletiva [Internet], 2000. Acesso em: 2017, set. 30, 5 (1): 163-177. Disponível em: <http://www.scielo.br/scielo. php?script=sci_arttext\&pid=S1413-81232000000100014\&lng=en. $\quad \underline{\text { http://dx.doi.org/10.1590/ }}$ $\underline{\text { S1413-81232000000100014>. }}$.

13. Minayo MCS, Hartz ZM, Buss PM. Qualidade de vida e saúde: um debate necessário. Ciênc. saúde coletiva [Internet], 2000. Acesso em: 2017, dez. 12, 5 (1): 7-18. Disponível em: http:// www.scielo.br/scielo.php?script=sci arttext\&pid=S1413-81232000000100002\&lng=en. http:// dx.doi.org/10.1590/S1413-81232000000100002.

14. Ursine BL, Trelha CS, Nunes EFPA. O Agente Comunitário de Saúde na Estratégia de Saúde da 
Família: uma investigação das condições de trabalho e da qualidade de vida. Rev. bras. saúde ocup. [Internet], 2010, dez. Acesso em: 2017, set. 30, 35 (122): 327-339. Disponível em: <http:// www.scielo.br/scielo.php?script=sci_arttext\&pid=S0303-76572010000200015\&lng=en. http:// dx.doi.org/10.1590/S0303-76572010000200015>.

15. Nahas MV. Atividade Física, saúde e qualidade de vida: conceitos e sugestões para um estilo de vida ativo, 6 ed. Londrina: Midiograf, 2013.

16. Siqueira CSV, Nahas MV, Facchini LA, Piccini RX, Tomasi E, Thumé E, et al. Atividade física em profissionais de saúde do Sul e Nordeste do Brasil. Cad Saúde Pública. Rio de Janeiro, 200. Acesso em: 28 set., 2019, 25 (9) 1917-1928. Disponível em: http://www.scielo.br/pdf/csp/ v25n9/06.pdf

17. Pizzolato BP, Moura, G. Silva AH. Qualidade de vida no trabalho: uma discussão sobre os modelos teóricos. Contribuciones a la Economía [Internet], 2013, abr. Acesso em: 2017, set. 20, 1-16. Disponível em: http://www.eumed.net/ce/2013/qualidade-vida-trabalho.html.

18. Figueiredo IM, Neves DS, Montanari D., Camelo SHH. Quality of life at work: perceptions of the Communitarian Agents of Health family teams. Rev enferm UERJ [Internet], 2009. Acesso em: 2017, set. 30], 17 (2): 262-7. Disponível em: http://www.facenf.uerj.br/v17n2/v17n2a22.pdf.

19. Mascarenhas CHM, Fernandes MH, Prado FO, Oliveira LL, Costa RFG, Santos DCL. Quality of community health agents. Arq Ciênc Saúde [Internet], 2012. Acesso em: 2017, set. 30, 19 (4): 97-103. Disponível em: <https://www.yumpu.com/pt/document/view/34230330/qualidade-de-vida-de-agentes-comunitarios-de-saade-life-quality-of- $>$.

20. Ximenes Neto FRG, Liberato BTG, Martins FR, Martins AR, Aguiar ERB. Qualidade de vida no trabalho dos trabalhadores da Estratégia Saúde da Família: um relato de experiência. Revista CPAQV [Internet], 2011. Acesso em: 2017, dez. 19, 3 (3): 1-6. Disponível em: http://www.cpaqv. org/revista/CPAQV/ojs-2.3.7/index.php?journal=CPAQV\&page=article\&op=view\&path\%5B $\% 5 \mathrm{D}=37$.

\section{DADOS DOS AUTORES}

\section{Francisco Rosemiro Guimarães Ximenes Neto}

Doutor em Ciências Universidade Federal de São Paulo (UNIFESP). Enfermeiro Sanitarista. Professor e Pesquisador do Curso de Enfermagem da Universidade Estadual Vale do Acaraú (UVA) e do Mestrado Profissional em Saúde da Família (UVA/RENASF/FIOCRUZ).rosemironeto@gmail.com

\section{Benedita Tatiane Gomes Liberato}

Acadêmica de Medicina Centro Universitário Inta (UNINTA). Sobral-Ceará. cariresaudavel@gmail. com

\section{Maria Roselange Guimarães Ximenes}

Acadêmica de Educação Física Centro Universitário Inta (UNINTA). Sobral-Ceará. roseximenes66@ gmail.com

\section{Francisco Diógenes dos Santos}

Enfermeiro. Especializando em Saúde Pública pela Escola de Saúde Pública do Ceará Paulo Marcelo Martins Rodrigues (ESP/CE). diogenezjunior@gmail.com

\section{Karla Mara Coelho Ponte de Oliveira}

Educadora Física. Mestranda em Saúde da Família pela UVA/RENASF/ FIOCRUZ. Coordenadora 
dos Núcleos de Apoio à Saúde da Família (NASF) e Academias da Saúde de Sobral-Ceará. kmaraponte@gmail.com

\section{Ana Gerúsia Souza Ribeiro Gurgel}

Enfermeira Sanitarista. Mestranda em Saúde da Família (UVA/RENASF/FIOCRUZ). Especialista em Gerência de Centros de Saúde da Família/UVA. Coordenadora da Atenção Primária de Sobral-Ceará. gerusiagurgel@gmail.com

Submetido em: 27-12-2017

Aceito em: 30-10-2019 